\title{
Gestational perturbation of homocysteine metabolism reduces Spina bifida prevalence in Osun State, Nigeria
}

\author{
Emmauel Olufemi Akanni'1, Adebayo Lawrence Adedeji², Oyindamola Nike Oyinlola', \\ Raufu Taiwo Azeez ${ }^{1}$, David Bolaji Akinbo ${ }^{3}$ \\ ${ }^{1}$ Haematology and Blood Transfusion Science Division, Department of Medical Laboratory Science, College of Health \\ Sciences, Ladoke Akintola University of Technology, P.M.B. 4400, Osogbo. Osun State, Nigeria, ${ }^{2}$ Department of \\ Biochemistry, College of Health Sciences, Ladoke Akintola University of Technology, P.M.B 4000, Ogbomosho; Oyo \\ State, Nigeria, ${ }^{3}$ Haematology and Blood Transfusion Science Division, Department of Medical Laboratory Science, \\ College of Medicine and Health Sciences, Afe Babalola University, P.M.B, 5454, Ado - Ekiti, Ekiti State, Nigeria.
}

Background: Elevated maternal homocysteine concentration has been described as a consequence of disruptions in methionine and/or folate metabolism which is associated with adverse outcomes and several obstetric complications inclusive of neural tube defects in offspring such as spina bifida, preeclampsia and spontaneous abortion. Aims and Objectives: The current study investigated the influence of maternal homocysteine metabolism in singleton pregnant women on the prevalence of Spina bifida in Osun State. Materials and Methods: Sixty (60) subjects attending Ladoke Akintola University of Technology Teaching Hospital and State Specialist Hospital in Osogbo, Osun State were randomly recruited for the study which spanned from January 2018 through April 2018 involving 30 apparently healthy singleton pregnant women classified according to gestational age and 30 apparently healthy age- and socio-economic status-matched non-pregnant women as controls. Haematocrit, haemoglobin concentration, total white cell count as well as the levels of homocysteine concentration were assessed. Results: Haematocrit and haemoglobin concentration in all three trimesters were significantly $(p<0.05)$ decreased when compared with their non-pregnant counterparts. There was however a significant $(p<0.05)$ increase in the expression of circulating homocysteine in all three trimesters compared to the control group, and an observed steady decline in the second trimester through the third trimester. Conclusion: These findings therefore imply that gestational perturbation of homocysteine metabolism may contribute to reduced risk of spina bifida in Osun State, Nigeria.

Key words: Homocysteine; Spina bifida; Singleton pregnancy; Folic acid;

Transmethylation

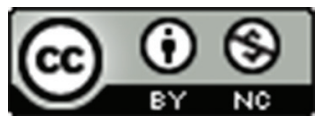

This work is licensed under a Creative Commons Attribution-NonCommercial 4.0 International License.

\section{INTRODUCTION}

Homocysteine (HCY) is a sulfur-containing amino acid and a metabolic intermediate in methyl group that is dependent on a number of nutritional B-vitamin cofactors. Compelling evidence have implicated the perturbations of homocysteine metabolism, particularly intracellular and subsequent circulation of accumulated homocysteine (hyperhomocysteinemia) in playing a key role in the etiology of disruption of neurodevelopment during fetal life, associated vascular disease risk as well as other pathologies. Several nutrient deficiencies are capable of giving rise to maternal hyperhomocysteinemia with folate being a prime candidate because folic acid deficient diets have been observed to restrict the recycling of homocysteine thereby limiting the supply of methyl groups used in a

Address for Correspondence:

David Bolaji Akinbo, Haematology and Blood Transfusion Science Division, Department of Medical Laboratory Science, College of Health

Sciences, Ladoke Akintola University of Technology, P.M.B. 4400Osogbo. Osun State, Nigeria. Telephone: +234 -7030-8577-29.

E-mail: dakinbo@abuad.edu.ng 
range of reactions essential for maternal metabolism and development of the foetus. ${ }^{1}$ This has culminated into recent insights on homocysteine metabolism in relation to health and disease conditions.

Pregnancy in humans has been associated with increased susceptibility to folate deficiency sequel to the elevated maternal requirement most especially in the later gestation. ${ }^{2}$ The unavailability or insufficiency of 5-MeTHF (5-Methyl Tetrahydrofolate) or cobalamin results in the accumulation of homocysteine suggestive of low folate concentrations being a marker for elevated homocysteine levels while previous studies have also reported a protective effect of the 5-MeTHF against homocysteine embryotoxicity in animal models. ${ }^{3}$ Previous studies have associated elevated concentrations of homocysteine in the maternal circulation with several obstetric complications inclusive of neural tube defects in offspring such as spina bifida (SB); which is a congenital malformation of the central nervous system, resulting from failure of the neural tube to close during early embryogenesis. ${ }^{2,4}$ Gestational hyperhomocysteinemia has been described as a consequence of distuptions in methionine and/or folate metabolism which has also been reported in adverse outcomes such as the neural tube defects, preeclampsia, spontaneous abortion, and premature delivery. ${ }^{3}$

Numerous studies have extensively examined the epidemiological characteristics of the Spina bifida describing the condition as the most common and complex central nervous system malformation in humans which occurs at the fourth week of post-fertilization. Spina bifida has an incidence rate of approximately 0.5 per 1,000 births varying among geographical regions worldwide, even within different locations of the same region and in different time periods; with prevalence in Nigeria varying between 0.28 $-0.5 \%$ and higher frequencies being recently reported. ${ }^{5,6}$ Previous works have emphatically demonstrated decreased levels of folate in the serum of women pregnant with a neural tube defect (NTD)-affected child during the first trimester. ${ }^{7}$ Similarly, recent studies have also reported adequate intake of folate to have regulated the biosynthesis of HCY during the periconceptional period and has been shown to reduce the possibility of SB development. ${ }^{8} \mathrm{SB}$ has reportedly constituted a major congenital malformation among neonatal referrals with an aggressive public health campaign being strongly advocated in order to enlighten women about the condition and encourage periconceptional folate supplementation., ${ }^{9,10}$ This study examined the association of maternal homocysteine metabolism in singleton pregnant women with the prevalence of Spina bifida in Osun State.

\section{MATERIALS AND METHODS}

This cross-sectional study was conducted among thirty (30) apparently healthy singleton pregnant women as confirmed by ultrasonography, who were attending Ladoke Akintola University of Technology Teaching Hospital and State Specialist Hospitals in Osogbo were randomly recruited for the study between January 2018 and April 2018 and classified according to their gestational age. The Ladoke Akintola University of Technology Teaching Hospital and State Specialist Hospitals are urban tertiary health institutions and major referral centers serving the whole of Osogbo and some other localities in Osun State. The control subjects were apparently healthy nonpregnant women from the vicinity of the hospitals and healthy women attending other specialist clinics such as the Dermatology Clinics; matched for age and socioeconomic status. Approval for the study was given by the Ethics Committee of the Ladoke Akintola University of Technology Teaching Hospital. Study information was made known to the potential participants in their first official languages and written informed consent was obtained from each participant.

None of the subjects or controls had a history of concomitant chronic illnesses like diabetes, evidence of cardiovascular, hepatic, renal, or gastrointestinal diseases or the presence of chronic infectious illnesses such as rhumatoid arthritis and AIDS. The selection criteria for the controls were the same as for study subjects and they had no symptoms or signs attributable to acute illness in the preceding four weeks. Demographic data were thereafter obtained for each participant. The study sample size consisted of 60 subjects comprising of 30 pregnant singleton women and 30 healthy non-pregnant women as control.

Five milliliters of blood were drawn from a convenient peripheral vein from each participant into vacuum tubes containing Ethylene Diamine Tetra-acetic acid (EDTA) and plain tubes, labeled and placed in a cool box containing ice-packs. The samples were protected from light at all times using sheets of black plastic, they were transported to the research laboratory of the Department of Medical Laboratory Science, Ladoke Akintola University of Technology Osogbo. The blood specimens were generally drawn in the morning, although the protocol did not require the subjects to be fasting; however, the effect of food intake on serum homocysteine levels is minimal as previously described. ${ }^{2}$ The blood specimens in the plain tubes were then centrifuged at $1000 \mathrm{rpm}$ for $15 \mathrm{~min}$; the serum separated and archived immediately at $-20^{\circ} \mathrm{C}$ in a single repository. All samples were handled and stored following a uniform, strict protocol. 
The hematological indices were assessed by flow cytometry (direct current method) using the automated hematologic auto-analyzer Sysmex, KXN21 (Japan) with the aid of suitable cell packs as earlier described. ${ }^{11,12}$ Plasma concentration of homocysteine (HCY) was determined by using human HCY ELISA kits according to the manufacturer's instructions (Hangzhou East Biopharm Co. Ltd, China). The data were expressed as mean \pm SEM in each group. Analysis of variance (ANOVA) was carried out to test for the level of homogeneity among study groups, while least square difference test was employed to determine the differences between means. All values were considered statistically significant at the level of $\mathrm{P}<0.05$.

\section{RESULTS}

Haematocrit and haemoglobin concentration in all three trimesters were significantly reduced $(p<0.05)$ in comparison to the control group with the lowest value occurring at the second trimester. The white blood cell counts in all the trimesters were significantly increased $(\mathrm{p}<0.05)$ compared to the normal control. The white blood cell counts were more significantly $(p<0.05)$ elevated in the third trimester group of the study than the other groups (Table 1).

In addition, the homocysteine levels were significantly increased $(p<0.05)$ in all three trimesters compared to the non-pregnant control group with those in their first semester having the highest homocysteine concentration. There was however, a significant $(\mathrm{p}<0.05)$ reduction in the circulating homocysteine levels of the second and third trimester groups respectively. The overall effects show that there was a significant accumulation of homocysteine at the early stage of the pregnancy period (Figure 1).

\section{DISCUSSION}

The key findings of the present study indicate that the haematocrit and haemoglobin concentration in all trimesters were lower than the nonpregnant controls, with an elevated total white blood cell count in the third trimester group than the first and second trimester's and the nonpregnant control. The plasma level of circulating homocysteine concentration was up-regulated in the first trimester and decreased to levels nearly comparable to those in the nonpregnant control group in the third trimester group. Pregnancy is characterized by many physiological hematological changes which may otherwise appear pathological in the non-pregnant state. As anticipated, the haematocrit and haemoglobin concentration during fasting were significantly lower in the pregnant subjects than the nonpregnant control group, and the differences for most of the haematocrit and haemoglobin concentration persisted all through the trimesters. The drop in haematocrit and haemoglobin concentration typically results from an increased plasma volume to red blood cell ratio. ${ }^{13}$ There is thus dilutional anaemia coexisting with macrocytes which may further result in a variety of disorders, this is consistent with previous reports by other studies. ${ }^{14,15}$ The primary mechanism of pregnancy-induced anaemia could therefore be related to the increased maternal requirement sequel to the increase in plasma volume particularly in later gestation which further fosters susceptibility to folate deficiency and the net result being a dip in the hemoglobin concentration. ${ }^{13,14}$

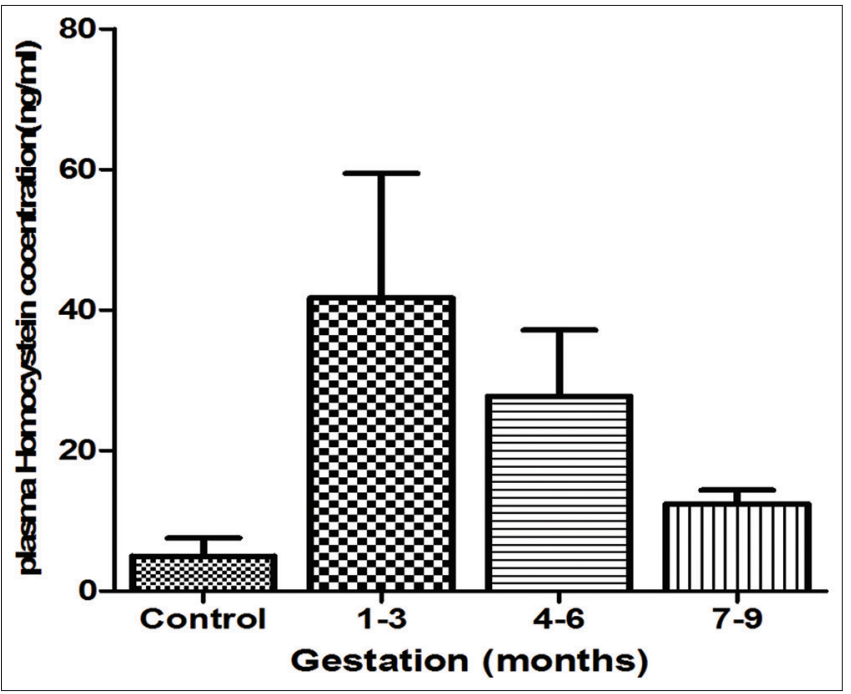

Figure 1: Plasma homocysteine variation in pregnant and non-pregnant subjects

\section{Table 1: Haematological indices and plasma homocysteine levels of the various groups}

\begin{tabular}{lcccc}
\hline & \multicolumn{4}{c}{ Variables } \\
\hline Group & PCV $(\%)$ & Hb. Conc. $(\mathbf{g} / \mathbf{d L})$ & WBC $\left(\times 10^{9} / \mathbf{L}\right)$ & HCY $(\mathbf{n m o l} / \mathbf{m L})$ \\
\hline First trimester & $32.75 \pm 2.49^{*}$ & $10.94 \pm 0.69^{*}$ & $5.95 \pm 1.23$ & $41.69 \pm 50.24^{*}$ \\
Second trimester & $30.90 \pm 2.07^{*}$ & $10.49 \pm 0.83^{*}$ & $6.69 \pm 2.81$ & $27.75 \pm 29.71^{* a}$ \\
Third trimester & $31.83 \pm 3.66^{*}$ & $10.60 \pm 1.23^{*}$ & $8.96 \pm 2.45^{\star a}$ & $12.40 \pm 6.73^{*}$ \\
Control & $36.67 \pm 2.06$ & $18.85 \pm 4.53$ & $3.99 \pm 1.051$ & $4.97 \pm 7.85$ \\
\hline
\end{tabular}

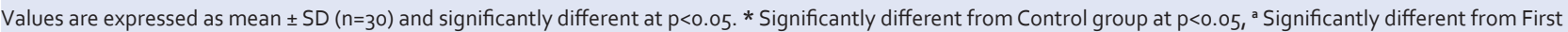
trimester group at $\mathrm{p}<0.05$. PCV: Packed Cell Volume, Hb. Conc.: Haemoglobin concentration, WBC: White blood cells, HCY: Homocysteine concentration 
In the present study, there was an elevated total leucocyte count with the lowest limit observed in the first trimester as consistent with other previous reports. ${ }^{16}$ Hematologic dyscrasias associated with pregnancy are well recognized and have been widely reported including reports of pregnancy-related leukocytosis from the second month of conception with an upward trend observed as pregnancy advances. ${ }^{17}$ Studies have reported infection and inflammation to have both caused excessive production of haemopoietic factors that act on the precursors of leukocytes and the megakaryocytes. ${ }^{18}$ Pregnancy is described as an inflammatory state with various changes in the immunological functions being generally initiated in order for the maternal body to accept and accommodate the fetal graft. ${ }^{19.20}$ The occurrence of leukocytosis during pregnancy can also result from the physiologic stress induced by the pregnant state and could be ascribed to an enhanced leucocyte production in the bone marrow in response to this stress as leucocyte levels have additionally been demonstrated to correlate with cervical dilation and labor progress. ${ }^{13}$

The significant increase in the homocysteine concentration in all three trimesters and the concomitant decline in the circulating levels from the second and third trimesters compared to the first trimester in this present study could be explained by the fact that cysteine and glutathione are provided for the fetus through the upregulation of methionine trans-sulfuration rate in the first trimester. ${ }^{21}$ The relevance of glutathione to the developing embryo has been suggested on the basis of data relating oxidant injury to the development of fetal malformations and a high rate of trans-sulfuration resulting in obligatory methionine requirement discovered to be consistent with high rate of NTDs in women with lower dietary intake of methionine. ${ }^{22,23}$ Clinical disorders such as eclampsia, spontaneous fetal loss, premature birth, birth defects, and fetal growth retardation associated with pregnancy have been described as major contributors to perinatal morbidities worldwide. Elevated total plasma homocysteine level has been recognized by previous studies as a risk factor for a number of adverse pregnancy outcomes in humans. ${ }^{15,24,25}$

A major consequence of reduced homocysteine methylation by folate and vitamin B12 deficiency is an elevated circulating level of homocysteine and formate in the plasma and urine. Similarly, folate, vitamins B12, B6 and B2 deficiencies have also been shown to result in increased maternal plasma homocysteine concentration which is capable of impacting fetal growth either directly by increased transport to the fetus or indirectly by impact on maternal health and pregnancy related disorders. ${ }^{26,27}$ Previous reports have shown that the supplementation of pregnant subjects with folic acid resulted in higher plasma and red blood cell folate levels and lower plasma homocysteine concentration. ${ }^{28}$ The decrease in transsulfuration during the third trimester can result in reduced plasma homocysteine concentrations in this trimester which may be due to supplementation of the pregnant women with folic acid subsequently causing lower levels of plasma homocysteine concentration as seen in the current study which is in consonance with other previous reports. ${ }^{21}$

Summarily, the present study revealed higher maternal plasma homocysteine level in the first trimester of pregnancy than the second and third trimesters' and in the nonpregnant state. However, the rate of transmethylation is higher in the third trimester than other trimesters and nonpregnancy state and this indicates the inhibition of trans-sulfuration pathway causing homocysteine disruption sequel to its sensitivity to folic acid availability which is possibly related to intervention with folate supplementation. Therefore, gestational perturbation of homocysteine metabolism may contribute to a reduced risk of spina bifida in Osun State, Nigeria.

\section{ACKNOWLEDGEMENTS}

The authors thank the authority of Ladoke Akintola University of Technology, Osogbo, Nigeria, for providing some of the facilities used in these studies. The technical assistance provided by Mrs. O. Olanrewaju of the Medical Laboratory Science department of College of Health Sciences, and the support by our research assistants is also appreciated.

\section{REFERENCES}

1. Rees WD, Wilson FA and Maloney CA. Sulfur amino acid metabolism in pregnancy: the impact of methionine in the maternal diet. J Nutr 2006; 36: 1701S-1705S.

https://doi.org/10.1093/jn/136.6.1701S

2. Brown SA, Bottiglieri T, Schaefer CA, Quesenberry Jr CP, Liu L, Bresnahan M, et al. Elevated Prenatal Homocysteine Levels as a Risk Factor for Schizophrenia. Arch Gen Psychiatry 2007; 64: 31-39. https://doi.org/10.1001/archpsyc.64.1.31

3. Imbard $A$, Benoist $\mathrm{J}$ and Blom HJ. Neural Tube Defects, Folic Acid and Methylation. Int J Environ Res Public Health 2013;10: 4352-4389.

https://doi.org/10.3390/ijerph10094352

4. Williams LJ, Rasmussen SA, Flores A, Kirby RS and Edmonds LD. Decline in the prevalence of spina bifida and anencephaly by race/ethnicity: 1995-2002. Pediatrics 2005; 116: 580-586. https://doi.org/10.1542/peds.2005-0592

5. Bulgakov OV, Eggenschwiler JT, Hong DH, Anderson KV, Li T. "FKBP8 is a negative regulator of mouse sonic hedgehog signaling in neural tissues." Development 2004;131(9): 2149-2159. https://doi.org/10.1242/dev.01122

6. Copp AJ and Greene NDE. "Neural tube defects-disorders of neurulation and related embryonic processes," Wiley Interdisciplinary 
Reviews: Developmental Biology 2013;2(2): 213-227.

https://doi.org/10.1002/wdev.71

7. Blom HJ and Smulders Y. Overview of homocysteine and folate metabolism. With special references to cardiovascular disease and neural tube defects. J Inherit Metab Dis 2011; 34(1): 75-81. https://doi.org/10.1007/s10545-010-9177-4

8. Bestwick JP, Huttly WJ, Morris JK and Wald NJ. Prevention of neural tube defects: a cross-sectional study of the uptake of folic acid supplementation in nearly half a million women. PLoS One 2014; 9: 89354

https://doi.org/10.1371/journal.pone.0089354

9. Ugwu RO, Eneh AU and Oruamabo RS. Neural tube defects in a University Teaching Hospital in Southern Nigeria: Trends and Outcome. Niger Journal Medicine 2007; 6(4): 368-371. https://doi.org/10.4314/njm.v16i4.37340

10. Rabiu TB, Tiamiyu LO and Awoyinka BS. Awareness of Spina Bifida and periconceptional use of folic acid among pregnant women in a developing economy. Child's Nervous System 2012; 28(12): 2115-2119.

https://doi.org/10.1007/s00381-012-1879-5

11. Akinbo $B D$, Atere $A D$ and Eluwole $O O$. Cytological Indices of Peripheral Blood and Bone Marrow Smears in Albino Rats infected with Trypanosoma brucei brucei. International Journal of Enhanced Research in Medicines \& Dental Care 2014;1(8): 7-11.

12. Akanni EO, Olaniran IO, Akinbo DB, Iyiola M, Ogunlade A, Sanni B, et al. Kigelia africana Stem Bark, Fruit and Leaf Extracts Alleviate Benzene-induced Leukaemia in Rats. JPRI 2017;18(2): 1-10.

https://doi.org/10.9734/JPRI/2017/34625

13. Akinlaja O. Hematological Changes in Pregnancy - The Preparation for Intrapartum Blood Loss. Obstet Gynecol Int J 2016; 4(3): 00109.

https://doi.org/10.15406/ogij.2016.04.00109

14. Chandra S, Tripathi AK, Mishra S, Amzarul M and Vaish AK. Physiological Changes in Hematological Parameters during Pregnancy. Indian J Hematol Blood Transfus 2012; 28(3): 144-146. https://doi.org/10.15406/ogij.2016.04.00109

15. Wilson FA, Holtrop G, Calder AG, Anderson SE, Lobley GE and Rees WD. Effects of methyl-deficient diets on methionine and homocysteine metabolism in the pregnant rat. American Journal of Physiology-Endocrinology and Metabolism 2012; 302(12): E1531-E1540.

https://doi.org/10.1152/ajpendo.00668.2011

16. Hossain $\mathrm{N}$ and Paidas MJ. Hematologic Changes in Pregnancy. Hemostasis and Thrombosis in Obstetrics \& Gynecology 2011;1-11. https://doi.org/10.1002/9781444328332.ch1

17. Pramanik SS, Pramanik T, Mondal SC and Chanda R. Number, maturity and phagocytic activity of neutrophils in the three trimesters of pregnancy. East Mediterr Health J 2007; 13(4): 862-867.
18. Thomas MR and Storey RF. The role of platelets in inflammation. Thrombosis and Haemostasis 2015; 114(3): 449-458.

https://doi.org/10.1160/TH14-12-1067

19. Anderson BL, Mendez-Figueroa H, Dahlke JD, Raker $\mathrm{C}$ and Hillier SL. Pregnancy-induced changes in immune protection of the genital tract: defining normal. Am J Obstet Gynecol 2013; 208(4): 321.E1-321.E9. https://doi.org/10.1016/j.ajog.2013.01.014

20. Redman CWG, Sargent IL and Taylor RN. 2014. Immunology of normal pregnancy and preeclampsia. In: Taylor R.N., et al. (Eds.), Chesley's Hypertensive Disorders in Pregnancy, (4th edn), Amsterdam Academic Press, Netherlands. https://doi.org/10.1016/B978-0-12-407866-6.00008-0

21. Dasarathy J, Gruca LL, Bennett C, Parimi PS, Duenas C, Marczewski $S$, et al. Methionine metabolism in human pregnancy. Am J Clin Nutr 2010. 91(2): 357-365. https://doi.org/10.3945/ajcn.2009.28457

22. Rees WD, Hay SM and Cruickshank M. An imbalance in the methionine content of the maternal diet reduces postnatal growth in the rat. Metabolism 2006;55: 763-770. https://doi.org/10.1016/j.metabol.2006.01.012

23. Loeken MR. Advances in understanding the molecular causes of diabetes-induced birth defects. J Soc Gynecol Investig 2006; 13: 2-10. https://doi.org/10.1016/j.jsgi.2005.09.007

24. Ueland PM and Vollset SE. Homocysteine and folate in pregnancy. Clin Chem 2004;50: 1293-1295. https://doi.org/10.1373/clinchem.2004.035709

25. Murphy MM, Scott JM, Arija V, Molloy AM and FernandezBallart JD. Maternal homocysteine before conception and throughout pregnancy predicts fetal homocysteine and birth weight. Clin Chem 2004;50: 1406-1412. https://doi.org/10.1373/clinchem.2004.032904

26. Barbosa PR, Stabler SP, Machado AL, Braga RC, Hirata RD, Hirata $\mathrm{MH}$, et al. Association between decreased vitamin levels and MTHFR, MTR and MTRR gene polymorphisms as determinants for elevated total homocysteine concentrations in pregnant women. Eur J Clin Nutr 2008; 62: 1010-1021.

https://doi.org/10.1038/sj.ejcn.1602810

27. Lamarre SG, Molloy AM, Reinke SN, Sykes BD, Brosnan ME and Brosnan JT. Formate can differentiate between hyperhomocysteinemia due to impaired remethylation and impaired transsulfuration. Am J Physiol Endocrinol Metab 2012; 302: E61-E67. https://doi.org/10.1152/ajpendo.00345.2011

28. Kalhan SC. One Carbon Metabolism in Pregnancy: Impact on Maternal, Fetal and Neonatal Health. Mol Cell Endocrinol 2016; 435: 48-60.

https://doi.org/10.1016/j.mce.2016.06.006

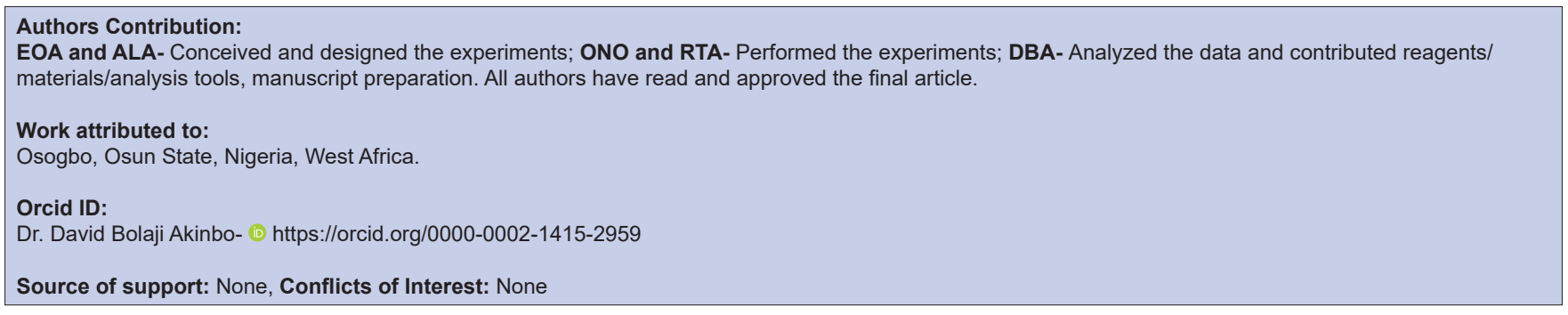

